\title{
SIGNATURE-BASED REPRESENTATIONS FOR THE RELIABILITY OF SYSTEMS WITH HETEROGENEOUS COMPONENTS
}

\author{
JORGE NAVARRO, ${ }^{*}$ Universidad de Murcia \\ FRANCISCO J. SAMANIEGO, ${ }^{* *}$ University of California, Davis \\ N. BALAKRISHNAN, ${ }^{* * *}$ McMaster University and King Saud University
}

\begin{abstract}
Signature-based representations of the reliability functions of coherent systems with independent and identically distributed component lifetimes have proven very useful in studying the ageing characteristics of such systems and in comparing the performance of different systems under varied criteria. In this paper we consider extensions of these results to systems with heterogeneous components. New representation theorems are established for both the case of components with independent lifetimes and the case of component lifetimes under specific forms of dependence. These representations may be used to compare the performance of systems with homogeneous and heterogeneous components.
\end{abstract}

Keywords: Coherent system; $k$-out-of- $n$ system; order statistic; signature; mixture; copula; stochastic order

2010 Mathematics Subject Classification: Primary 60E15

Secondary $60 \mathrm{~K} 10$

\section{Introduction}

The notion of system signatures was introduced by Samaniego [25] as a useful tool in providing a characterization of the class of coherent systems which satisfy the IFR (increasing failure rate) closure property for systems with independent and identically distributed (i.i.d.) component lifetimes. The signature of a coherent system whose $n$ components have i.i.d. lifetimes was defined as the $n$-dimensional probability vector whose $i$ th element is $s_{i}=\mathrm{P}(T=$ $\left.X_{i: n}\right)$, where $T$ denotes the lifetime of the system and $\left(X_{1: n}, \ldots, X_{n: n}\right)$ is the vector of order statistics of the component lifetimes $X_{1}, \ldots, X_{n} \sim F$. The distribution $F$ is assumed to be continuous with support set $(0, \infty)$. Under the i.i.d. assumption on component lifetimes, the signature vector $s=\left(s_{1}, \ldots, s_{n}\right)$ is a distribution-free measure of the system's design. The system's reliability function $\bar{F}_{T}(t)=\mathrm{P}(T>t)$ may be represented as

$$
\bar{F}_{T}(t)=\sum_{i=1}^{n} s_{i} \bar{F}_{i: n}(t),
$$

\footnotetext{
Received 6 January 2011; revision received 14 March 2011.

* Postal address: Facultad de Matemáticas, Universidad de Murcia, 30100 Murcia, Spain.

Email address: jorgenav@um.es

** Postal address: Department of Statistics, University of California, Davis, 1 Shields Avenue, 95616 Davis, CA, USA. Email address: fjsamaniego@ucdavis.edu

*** Postal address: Department of Mathematics and Statistics, McMaster University, Hamilton, Ontario L8S 4K1, Canada. Email address: bala@mcmaster.ca
} 
where $\bar{F}_{i: n}(t)=\mathrm{P}\left(X_{i: n}>t\right)$. The monograph by Samaniego [26] provides an extensive survey of system signatures and their applications.

Since (1.1) may also be written as

$$
\bar{F}_{T}(t)=\sum_{i=1}^{n} s_{i} \sum_{j=n-i+1}^{n}\left(\begin{array}{l}
n \\
j
\end{array}\right)\{\bar{F}(t)\}^{j}\{F(t)\}^{n-j},
$$

we may express the reliability of the system at time $t$ through the reliability polynomial $h(p)$ (see [2, p. 21]) given by

$$
h(p)=\sum_{i=1}^{n} s_{i} \sum_{j=n-i+1}^{n}\left(\begin{array}{l}
n \\
j
\end{array}\right) p^{j}(1-p)^{n-j},
$$

where $p=\bar{F}(t)$. This polynomial may also be written in the form $h(p)=\sum_{i=1}^{n} d_{i} p^{i}$, where $\boldsymbol{d}=\left(d_{1}, \ldots, d_{n}\right)$ is generally called the system's domination vector (see [27]). Explicit formulae for the relationships $\boldsymbol{d}=g(\boldsymbol{s})$ and $s=g^{-1}(\boldsymbol{d})$ connecting the signature and domination vectors may be found in [26]. In the sequel, we will utilize the reliability polynomial $h(p)$ primarily in the form (1.2). The coefficients of this polynomial depend only on the system structure. The polynomial $h(p)$ is strictly increasing for $p \in(0,1)$, with $h(0)=0$ and $h(1)=1$.

The representation in (1.1) continues to hold when the components are dependent with a joint exchangeable distribution; see [22] and the references therein. It also holds for mixed systems, that is, for stochastic mixtures of coherent systems (see [26]). However, this representation is not necessarily true when the component lifetimes have different distributions, as demonstrated in Example 5.1 of [22].

Other useful representations for coherent systems with exchangeable components are

$$
\bar{F}_{T}(t)=\sum_{i=1}^{n} a_{i} \bar{F}_{1: i}(t)
$$

and

$$
\bar{F}_{T}(t)=\sum_{i=1}^{n} b_{i} \bar{F}_{i: i}(t),
$$

where $\bar{F}_{1: i}(t)=\mathrm{P}\left(X_{1: i}>t\right)$ and $\bar{F}_{i: i}(t)=\mathrm{P}\left(X_{i: i}>t\right)$ are the reliability functions of the series system lifetime $X_{1: i}=\min \left(X_{1}, \ldots, X_{i}\right)$ and the parallel system lifetime $X_{i: i}=$ $\max \left(X_{1}, \ldots, X_{i}\right)$, respectively. The vectors of coefficients $\boldsymbol{a}=\left(a_{1}, \ldots, a_{n}\right)$ and $\boldsymbol{b}=$ $\left(b_{1}, \ldots, b_{n}\right)$, which depend only on the system structure, are called minimal and maximal signatures, respectively; see [19]. While some of the elements of $\boldsymbol{a}$ and $\boldsymbol{b}$ may be negative, the vectors obey the constraints $\sum_{i=1}^{n} a_{i}=1$ and $\sum_{i=1}^{n} b_{i}=1$, respectively. When the component lifetimes are i.i.d., representation (1.3) reduces to

$$
\bar{F}_{T}(t)=\sum_{i=1}^{n} a_{i}\{\bar{F}(t)\}^{i}
$$

where $\bar{F}$ is the common reliability function of the components. However, representations (1.3) and (1.4) do not necessarily hold when the components have different distributions, as 
demonstrated in Example 5.1 of [22]. Related work on system signatures includes [4], [5], [9], [10], [11], [14], [15], [20], [29], and [30].

Few representation results have been obtained in the literature for coherent systems with heterogeneous components. Generalized mixture representations based on minimal path sets and minimal cut sets were obtained by Esary and Proschan [7] (see also [2, p. 26]) in the case of independent components. Also, in this case, some comparison results were obtained in [14] and [21]. In the case of dependent heterogeneous components, representations and bounds for $k$-out-of- $n$ systems (order statistics) were obtained in [24, Chapter 5], and comparisons between parallel and series systems were given in [17] and [18].

In this paper, representations similar to those in (1.1), (1.3), and (1.4) are obtained for coherent systems with heterogeneous components. We consider the case of independent components in Section 2 and the case of dependent components in Section 3. These representations allow us to compare the performance of a given system under differing assumptions on component lifetimes-i.i.d. lifetimes and independent but heterogeneous lifetimes. We also use these representations to obtain some ordering properties.

Throughout the paper, when we say that a function $g$ is increasing or decreasing, we mean that $g(x) \leq g(y)$ or $g(x) \geq g(y)$ for $x \leq y$, respectively.

\section{Signature representations for systems with independent components}

Let $T=\phi\left(X_{1}, \ldots, X_{n}\right)$ be the lifetime of a coherent system with independent component lifetimes $X_{1}, \ldots, X_{n}$ with respective reliability functions $\bar{F}_{1}, \ldots, \bar{F}_{n}$, each assumed to be continuous and with supports contained in $(0, \infty)$, where $\phi$ is the structure function of the system (see [2]). If $X_{1: n}<\cdots<X_{n: n}$ are the order statistics obtained from $X_{1}, \ldots, X_{n}$ then it is evident that $\mathrm{P}\left(T=X_{i: n}\right)$ depends on the distributions of $X_{1}, \ldots, X_{n}$. While the reliability function of $T$ may still be written as a sum similar to that in (1.1), that is, as

$$
\bar{F}_{T}(t)=\sum_{i=1}^{n} \mathrm{P}\left(T=X_{i: n}\right) \mathrm{P}\left(X_{i: n}>t \mid T=X_{i: n}\right),
$$

this representation is not useful in the analysis and comparisons of systems due to the dependence of $\mathrm{P}\left(T=X_{i: n}\right)$ and $\mathrm{P}\left(X_{i: n}>t \mid T=X_{i: n}\right)$ on both $\left\{\bar{F}_{i}, i=1, \ldots, n\right\}$ and $\phi$. In what follows, we will develop an alternative representation of $\bar{F}_{T}$ which, quite unexpectedly, relies on the system's distribution-free signature vector $s$ (generally defined under an i.i.d. assumption on component lifetimes) defined in the previous section, notwithstanding the fact that the system under study is assumed to have heterogeneous rather than identical components. Under the i.i.d. assumption on component lifetimes, the signature of a coherent system does not depend on the common component lifetime distribution and may be computed as $s_{i}=\left|A_{i}\right| / n$ ! for $i=1, \ldots, n$, where $\left|A_{i}\right|$ is the cardinality of the set $A_{i}$ of permutations $\sigma$ of the set $\{1, \ldots, n\}$ such that $\phi\left(X_{1}, \ldots, X_{n}\right)=X_{i: n}$ whenever $X_{\sigma(1)}<\cdots<X_{\sigma(n)}$ (see [26]).

If a coherent system with lifetime $T$ has independent components then its reliability function can be written as

$$
\bar{F}_{T}(t)=H\left(\bar{F}_{1}(t), \ldots, \bar{F}_{n}(t)\right),
$$

where $H\left(p_{1}, \ldots, p_{n}\right)$ is a multinomial expression (called the structure reliability function in [7]) which is linear in each $p_{i}$ and whose coefficients depend only on the system's design. In analogy with (1.2), at a fixed time $t$, the reliability of the system at time $t$ may be written as $H\left(p_{1}, \ldots, p_{n}\right)$, where $p_{i}=\bar{F}_{i}(t)$ for $i=1, \ldots, n$. This polynomial is strictly increasing 
in $(0,1)^{n}$ and is such that $H(0, \ldots, 0)=0$ and $H(1, \ldots, 1)=1$. Clearly, the reliability polynomial in (1.2) is simply $h(p)=H(p, \ldots, p)$. Using representation (2.1), we can extend representation (1.1) as follows.

As defined above, the signature of a coherent system is a 'topological invariant' which can be treated without reference to the stochastic behavior of the system's components. While it is generally defined in terms of the order statistics of an i.i.d. sample from a common component distribution $F$, the signature vector depends solely on the system's design and does not depend on the distribution of component lifetimes. For example, the signature vectors of all fourcomponent systems are displayed in [26] (see also [14] and [29]). It is true that the application and interpretation of system signatures have largely been restricted to the case of systems with i.i.d. component lifetimes. For example, the representation of a system's reliability function in terms of the system's signature, as in (1.1), requires the assumption of i.i.d. component lifetimes. The following result shows that the signature of a given system may be used in an analogous fashion in representing the reliability function of the system when component lifetimes are independent but not homogeneous. The representation is seen to involve a single distribution $G$ that is functionally dependent on the individual lifetime distributions of the system's components as well as on the system's design.

Theorem 2.1. Consider an $n$-component system with signature vector $s=\left(s_{1}, \ldots, s_{n}\right)$, and let $h$ and $H$ be the system's reliability polynomial and structure reliability function, respectively. Suppose that the lifetimes $X_{1}, \ldots, X_{n}$ of the components of this system are independent but are not identically distributed, with $X_{i}$ having the continuous distribution function $F_{i}$ and reliability function $\bar{F}_{i}=1-F_{i}$ for $i=1, \ldots, n$. Then the reliability function of the system lifetime $T$ may be expressed as

$$
\bar{F}_{T}(t)=\sum_{i=1}^{n} s_{i} \bar{G}_{i: n}(t),
$$

where $\bar{G}_{i: n}(t)=\mathrm{P}\left(Y_{i: n}>t\right)$ and $Y_{1: n}<\cdots<Y_{n: n}$ are the order statistics obtained from the i.i.d. random variables $Y_{1}, \ldots, Y_{n}$ with common reliability function

$$
\bar{G}(t)=h^{-1}\left(H\left(\bar{F}_{1}(t), \ldots, \bar{F}_{n}(t)\right)\right) .
$$

Proof. Recall that the reliability polynomial $h$ is a strictly increasing continuous function in $(0,1)$ with $h(0)=0$ and $h(1)=1$. Hence, its inverse function $h^{-1}$ in $(0,1)$ exists and is also strictly increasing in $(0,1)$ with $h^{-1}(0)=0$ and $h^{-1}(1)=1$. We also know that $H$ is a strictly increasing continuous function in $(0,1)^{n}$ with $H(0, \ldots, 0)=0$ and $H(1, \ldots, 1)=1$. Hence, the function $\bar{G}$ defined by (2.3) is right continuous and decreasing in $t$ with $\bar{G}(0)=1$ and $\bar{G}(\infty)=0$, and is therefore a proper reliability function. If a system with signature $s$ has components with i.i.d. lifetimes with the common distribution $G$ whose reliability function is as given in (2.3), then it follows from (1.2) that the system's reliability function is given by $h(\bar{G}(t))$. We thus find from $(1.1)$ that this reliability function may be represented as

$$
h(\bar{G}(t))=\sum_{i=1}^{n} s_{i} \bar{G}_{i: n}(t) .
$$

Finally, we note from (2.3) that we have

$$
h(\bar{G}(t))=H\left(\bar{F}_{1}(t), \ldots, \bar{F}_{n}(t)\right),
$$

and, hence, due to (2.1), (2.2) holds. 
This theorem can be used to obtain ordering properties similar to those given in [11] and [22], but for systems with heterogeneous components. We consider the following stochastic orders. Let $X$ and $Y$ be two random variables with respective absolutely continuous distribution functions $F$ and $G$, hazard rate functions $r_{F}=F^{\prime} /(1-F)$ and $r_{G}=G^{\prime} /(1-G)$, and reversed hazard rate functions $\bar{r}_{F}=F^{\prime} / F$ and $\bar{r}_{G}=G^{\prime} / G$. Then the following statements hold:

(i) $X$ is said to be less than $Y$ in the stochastic order (written as $X \leq_{\text {st }} Y$ ) when $F \geq G$;

(ii) $X$ is said to be less than $Y$ in the hazard rate order (written as $X \leq{ }_{\mathrm{hr}} Y$ ) when $r_{F} \geq r_{G}$;

(iii) $X$ is said to be less than $Y$ in the reversed hazard rate order (written as $X \leq_{\mathrm{rh}} Y$ ) when $\bar{r}_{F} \leq \bar{r}_{G}$.

The main properties of these orders can be found in [28, Chapter 1]. To obtain our ordering results, we need the following lemma, which is a direct consequence of Theorems 4.5 and 5.5 of [3].

Lemma 2.1. Let $T$ and $T^{*}$ be the lifetimes of two coherent systems with the same structure, reliability polynomial $h$, and i.i.d. components having common reliability functions $\bar{F}$ and $\bar{F}^{*}$, respectively.

(i) If $\bar{F} \leq_{\mathrm{hr}} \bar{F}^{*}$ and $x h^{\prime}(x) / h(x)$ is decreasing, then $T \leq_{\mathrm{hr}} T^{*}$.

(ii) If $\bar{F} \leq_{\mathrm{rh}} \bar{F}^{*}$ and $(1-x) h^{\prime}(x) /(1-h(x))$ is increasing, then $T \leq_{\mathrm{rh}} T^{*}$.

Theorem 2.2. Let $T$ and $T^{*}$ be the lifetimes of two coherent systems with signatures $\boldsymbol{s}=$ $\left(s_{1}, \ldots, s_{n}\right)$ and $s^{*}=\left(s_{1}^{*}, \ldots, s_{n}^{*}\right)$, and both with independent component lifetimes. Let $h$ and $h^{*}$ be their reliability polynomials, and let $H$ and $H^{*}$ be their structure reliability functions. Let $\bar{G}$ and $\bar{G}^{*}$ be the reliability functions defined in (2.3).

(i) If $\bar{G} \leq_{\mathrm{st}} \bar{G}^{*}$ and $\mathbf{s} \leq_{\mathrm{st}} \boldsymbol{s}^{*}$, then $T \leq_{\mathrm{st}} T^{*}$.

(ii) If $\bar{G} \leq_{\mathrm{hr}} \bar{G}^{*}, \mathbf{s} \leq \mathrm{hr} s^{*}$, and either $x h^{\prime}(x) / h(x)$ or $x\left(h^{*}\right)^{\prime}(x) / h(x)$ is decreasing, then $T \leq \mathrm{hr} T^{*}$.

(iii) If $\bar{G} \leq_{\mathrm{rh}} \bar{G}^{*}, \mathbf{s} \leq_{\mathrm{rh}} \boldsymbol{s}^{*}$, and either $(1-x) h^{\prime}(x) /(1-h(x))$ or $(1-x)\left(h^{*}\right)^{\prime}(x) /\left(1-h^{*}(x)\right)$ is increasing, then $T \leq_{\mathrm{rh}} T^{*}$.

Proof. The proof of (i) is immediate from Theorem 2.1 .

We prove (ii) by assuming that $x h^{\prime}(x) / h(x)$ is increasing. The proof of the other case is similar. Then we have

$$
\begin{aligned}
\bar{F}_{T}(t) & =\sum_{i=1}^{n} s_{i} \bar{G}_{i: n}(t) \quad(\text { from Theorem } 2.1) \\
& \leq \mathrm{hr} \sum_{i=1}^{n} s_{i} \bar{G}_{i: n}^{*}(t) \quad\left(\text { from } \bar{G} \leq_{\mathrm{hr}} \bar{G}^{*} \text { and Lemma } 2.1\right) \\
& \leq \mathrm{hr} \sum_{i=1}^{n} s_{i}^{*} \bar{G}_{i: n}^{*}(t) \quad\left(\text { from } s \leq_{\mathrm{hr}} s^{*}\right) \\
& =\bar{F}_{T^{*}(t) \quad(\text { from Theorem } 2.1)}
\end{aligned}
$$

where the last inequality holds by Theorem 1.B.14 of [28]. 
The proof of (iii) can be obtained in a similar way by using Lemma 2.1(ii) and Theorem 1.B.52 of [28].

Example 2.1. If we consider the coherent system with lifetime $T=\max \left(\min \left(X_{1}, X_{2}\right)\right.$, $\min \left(X_{3}, X_{4}\right)$ ) and corresponding signature $s=\left(0, \frac{2}{3}, \frac{1}{3}, 0\right)$ (see system number 10 in Table 3.2 of $[26$, p. 25]), then its reliability function is given by

$$
\begin{aligned}
\bar{F}_{T}(t) & =\mathrm{P}\left(\max \left(\min \left(X_{1}, X_{2}\right), \min \left(X_{3}, X_{4}\right)\right)>t\right) \\
& =\mathrm{P}\left(\left\{\min \left(X_{1}, X_{2}\right)>t\right\} \cup\left\{\min \left(X_{3}, X_{4}\right)>t\right\}\right) \\
& =\mathrm{P}\left(\min \left(X_{1}, X_{2}\right)>t\right)+\mathrm{P}\left(\min \left(X_{3}, X_{4}\right)>t\right)-\mathrm{P}\left(X_{1: 4}>t\right) \\
& =\bar{F}_{1}(t) \bar{F}_{2}(t)+\bar{F}_{3}(t) \bar{F}_{4}(t)-\bar{F}_{1}(t) \bar{F}_{2}(t) \bar{F}_{3}(t) \bar{F}_{4}(t) \\
& =H\left(\bar{F}_{1}(t), \bar{F}_{2}(t), \bar{F}_{3}(t), \bar{F}_{4}(t)\right),
\end{aligned}
$$

where $H\left(p_{1}, p_{2}, p_{3}, p_{4}\right)=p_{1} p_{2}+p_{3} p_{4}-p_{1} p_{2} p_{3} p_{4}$. Also, $h(p)=2 p^{2}-p^{4}$ and its minimal signature is $\boldsymbol{a}=(0,2,0,-1)$. Then,

$$
h^{-1}(x)=\sqrt{1-\sqrt{1-x}}
$$

and the reliability function defined in (2.3) is

$$
\bar{G}(t)=\sqrt{1-\sqrt{1-\bar{F}_{1}(t) \bar{F}_{2}(t)-\bar{F}_{3}(t) \bar{F}_{4}(t)+\bar{F}_{1}(t) \bar{F}_{2}(t) \bar{F}_{3}(t) \bar{F}_{4}(t)}} .
$$

In general, we know that $h(p)$ is a strictly increasing polynomial in $p \in[0,1]$ from $h(0)=0$ and $h(1)=1$, and, hence, its inverse function $h^{-1}(x)$ exists for $x \in[0,1]$. However, the explicit computation of $h^{-1}(x)$ is not always as easy as in the preceding example. In general, numerical methods must be employed to obtain an approximation of $\bar{G}$.

Note that the reliability function $\bar{G}$ in (2.3) is a function such that

$$
H(\bar{G}(t), \ldots, \bar{G}(t))=H\left(\bar{F}_{1}(t), \ldots, \bar{F}_{n}(t)\right) .
$$

This reliability function is related to the well-known concept of mean function associated with a real-valued function (see [6], [8, p. 65], and [18]), which is defined as follows.

Definition 2.1. If $\psi: S \subseteq \mathbb{R}^{n} \rightarrow \mathbb{R}$ is a real-valued function, a mean function of $\psi$ in $S$ is a function $m_{\psi}: \mathbb{R}^{n} \rightarrow \mathbb{R}$ such that

$$
\psi\left(x_{1}, \ldots, x_{n}\right)=\psi(z, \ldots, z)
$$

for all $\left(x_{1}, \ldots, x_{n}\right) \in S$, where $z=m_{\psi}\left(x_{1}, \ldots, x_{n}\right)$.

Clearly, a mean function of $\psi$ exists when its diagonal section $\delta(z)=\psi(z, \ldots, z)$ is continuous, $\inf _{\boldsymbol{x} \in S} \psi(\boldsymbol{x})=\inf _{z \in D} \delta(z)$ and $\sup _{\boldsymbol{x} \in S} \psi(\boldsymbol{x})=\sup _{z \in D} \delta(z)$, where $D=\{z \in$ $\mathbb{R}:(z, \ldots, z) \in S\}$. Moreover, the mean function is unique when the preceding properties hold and $\delta(z)$ is strictly monotone in $D$. For example, if $\psi\left(x_{1}, x_{2}, x_{3}\right)=x_{1} x_{2} x_{3}$ then $\delta(z)=z^{3}$ and its unique mean function in $\mathbb{R}^{3}$ is $m_{\psi}\left(x_{1}, x_{2}, x_{3}\right)=\left(x_{1} x_{2} x_{3}\right)^{1 / 3}$. However, the mean function in $\mathbb{R}^{2}$ of $\psi\left(x_{1}, x_{2}\right)=x_{1} x_{2}$ does not exist.

Note that the mean function $m_{H}$ in $[0,1]^{n}$ of the structure reliability function $H$ of an $n$-component system always exists since its diagonal section $h(z)=H(z, \ldots, z)$ is a strictly increasing polynomial in $[0,1]$ with $h(0)=0$ and $h(1)=1$. Moreover, it is given by

$$
m_{H}\left(x_{1}, \ldots, x_{n}\right)=h^{-1}\left(H\left(x_{1}, \ldots, x_{n}\right)\right)
$$


and satisfies $m_{H}(z, \ldots, z)=z$. Hence, the reliability function defined in (2.3) satisfies

$$
\bar{G}(t)=m_{H}\left(\bar{F}_{1}(t), \ldots, \bar{F}_{n}(t)\right) .
$$

Thus, this reliability function $\bar{G}(t)$ can appropriately be called the mean reliability function associated with the system and the component reliability functions.

For example, in the case of a series system, the mean function of $H$ is the geometric mean

$$
m_{H}\left(x_{1}, \ldots, x_{n}\right)=\left(x_{1} \cdots x_{n}\right)^{1 / n} .
$$

For the parallel system, the mean function is

$$
m_{H}\left(x_{1}, \ldots, x_{n}\right)=1-\left[\left(1-x_{1}\right) \cdots\left(1-x_{n}\right)\right]^{1 / n} .
$$

For the system considered in Example 2.1, the mean function is

$$
m_{H}\left(x_{1}, x_{2}, x_{3}, x_{4}\right)=\sqrt{1-\sqrt{1-x_{1} x_{2}-x_{3} x_{4}+x_{1} x_{2} x_{3} x_{4}}},
$$

and its mean reliability function is as given in (2.4).

Note that, from Theorem 2.2, the mean reliability function defined in (2.3) provides a vehicle for comparing the performance of a system whose components have i.i.d. lifetimes with distribution $F$ and the same system with components whose lifetimes are independent but have heterogeneous distributions.

Next, we extend representations (1.3) and (1.4) to systems with heterogeneous components. The result is given in the following theorem and it is an immediate consequence of Theorem 2.1. First, we need to extend the definition of minimal and maximal signatures to systems with heterogeneous components as follows. If $T$ is the lifetime of a coherent system with independent components, we define the minimal and maximal signatures $\boldsymbol{a}=\left(a_{1}, \ldots, a_{n}\right)$ and $\boldsymbol{b}=$ $\left(b_{1}, \ldots, b_{n}\right)$ of $T$ as the minimal and maximal signatures of a system with the same structure as $T$ but with i.i.d. component lifetimes with common reliability function $\bar{G}(t)$. It is useful to note once again that these signatures do not depend on the component distributions and that they can be obtained from the system signature $s=\left(s_{1}, \ldots, s_{n}\right)$.

Theorem 2.3. If $T$ is the lifetime of a coherent system with independent component lifetimes $X_{1}, \ldots, X_{n}$, and minimal and maximal signatures $\boldsymbol{a}=\left(a_{1}, \ldots, a_{n}\right)$ and $\boldsymbol{b}=\left(b_{1}, \ldots, b_{n}\right)$, then

$$
\bar{F}_{T}(t)=\sum_{i=1}^{n} a_{i} \bar{G}_{1: i}(t)
$$

and

$$
\bar{F}_{T}(t)=\sum_{i=1}^{n} b_{i} \bar{G}_{i: i}(t),
$$

where $\bar{G}_{1: i}(t)=\bar{G}^{i}(t)$ and $\bar{G}_{i: i}(t)=1-G^{i}(t)$ for $i=1, \ldots, n, \bar{G}$ is as given in (2.3), and $G=1-\bar{G}$.

Let us consider an application of this theorem. Since the reliability polynomial may be written as $h(p)=\sum_{i=1}^{n} a_{i} p^{i},(2.5)$ can alternatively be expressed as

$$
\bar{F}_{T}(t)=\sum_{i=1}^{n} a_{i}\{\bar{G}(t)\}^{i} .
$$


Recall that the coefficients $a_{i}$ are real numbers such that $\sum_{i=1}^{n} a_{i}=1$ and, therefore, $\bar{F}_{T}$ is a generalized mixture. Then, (2.6) shows that the class of all reliability functions of coherent systems with $n$ independent components is included in the class of generalized mixtures obtained from powers $\bar{G}^{i}$ of a reliability function $\bar{G}$ for $i=1, \ldots, n$. Hence, the asymptotic behavior (when $t \rightarrow \infty$ ) of the ageing measures of $T$ can be determined from the results established in [12], [13], and [16]. For example, from the results in [16], it is known that if the components are independent then the hazard rate function $r_{T}$ of the system satisfies

$$
\lim _{t \rightarrow \infty} \frac{r_{T}(t)}{r_{G}(t)}=i
$$

where $r_{G}$ is the hazard rate function associated with $G=1-\bar{G}$ and $i=\min \left\{j: a_{j} \neq 0\right\}$.

\section{Signature representations for systems with dependent components}

In this section we extend the representations based on signatures given in the preceding section to coherent systems with component lifetimes that may be dependent. Let $T=$ $\phi\left(X_{1}, \ldots, X_{n}\right)$ be the lifetime of a coherent system with structure function $\phi$ and with component lifetimes $\left(X_{1}, \ldots, X_{n}\right)$. The dependence between component lifetimes can be represented by the joint reliability (or survival) function

$$
\overline{\boldsymbol{F}}\left(x_{1}, \ldots, x_{n}\right)=\mathrm{P}\left(X_{1}>x_{1}, \ldots, X_{n}>x_{n}\right) .
$$

Using Sklar's celebrated theorem (see [23, p. 46]), this reliability function can be written as

$$
\overline{\boldsymbol{F}}\left(x_{1}, \ldots, x_{n}\right)=K\left(\bar{F}_{1}\left(x_{1}\right), \ldots, \bar{F}_{n}\left(x_{n}\right)\right),
$$

where $\bar{F}_{i}$ is the reliability function of $X_{i}$ and $K$ is the survival copula. It is well known that the survival copula can be obtained from the distributional copula (i.e., the copula used to represent the joint distribution function) and vice versa.

For our purposes, we will also need the well-known representation of the lifetime of a coherent system based on its minimal path sets (see [2, p. 12]). A set $P \subseteq\{1, \ldots, n\}$ is a path set of a coherent system if the system works when all the components in $P$ work. A path set $P$ is a minimal path set if it does not contain other path sets. If $T$ is the lifetime of a coherent system with minimal path sets $P_{1}, \ldots, P_{k}$, then

$$
T=\max _{j=1, \ldots, k} X_{P_{j}}
$$

where $X_{P_{j}}=\min _{i \in P_{j}} X_{i}$ is the lifetime of the series system with components in $P_{j}$ for $j=1, \ldots, k$ (see [2, p. 12]). Hence, it follows from the inclusion-exclusion formula (see [1] and [19]) that the reliability function of the system can be expressed as

$$
\bar{F}_{T}(t)=\sum_{j=1}^{k} \bar{F}_{P_{j}}(t)-\sum_{i<j} \bar{F}_{P_{i} \cup P_{j}}(t)+\cdots+(-1)^{k+1} \bar{F}_{P_{1} \cup \cdots \cup P_{k}}(t),
$$

where $\bar{F}_{P}$ is the reliability function of the series system lifetime $X_{P}=\min _{i \in P} X_{i}$. Note that $P_{1} \cup \cdots \cup P_{k}=\{1, \ldots, n\}$. Furthermore, $\bar{F}_{P}$ can be written as

$$
\bar{F}_{P}(t)=\bar{F}\left(t_{P}\right),
$$

where $\boldsymbol{t}_{P}=\left(x_{1}, \ldots, x_{n}\right)$ with $x_{i}=t$ for $i \in P$ and $x_{i}=0$ for $i \notin P$. Taking into account this 
representation for series system reliability functions and using Sklar's representation (3.1) for the survival copula, (3.2) can be written as

$$
\bar{F}_{T}(t)=W\left(\bar{F}_{1}(t), \ldots, \bar{F}_{n}(t)\right),
$$

where

$$
W\left(x_{1}, \ldots, x_{n}\right)=\sum_{j=1}^{k} K\left(\boldsymbol{x}_{P_{j}}\right)-\sum_{i<j} K\left(\boldsymbol{x}_{P_{i} \cup P_{j}}\right)+\cdots+(-1)^{k+1} K\left(\boldsymbol{x}_{P_{1} \cup \ldots \cup P_{k}}\right)
$$

and $\boldsymbol{x}_{P}=\left(z_{1}, \ldots, z_{n}\right)$ with $z_{i}=x_{i}$ for $i \in P$ and $z_{i}=1$ for $i \notin P$. In particular, if the components are independent then the function $W$ is equal to the structure reliability function $H$. If the system is a series system then $W$ is equal to the survival copula $K$. However, in general, $W$ is neither a polynomial nor a copula and it depends both on the system structure and on the survival copula (i.e., the dependence structure between the components). However, it should be noted that $W$ does not depend on the component (marginal) distributions. We will refer to the function $W=W(\phi, K)$ as the structure-dependence function.

For example, let us consider the system with lifetime $T=\min \left(X_{1}, \max \left(X_{2}, X_{3}\right)\right)$ with minimal path sets $P_{1}=\{1,2\}$ and $P_{2}=\{1,3\}$. Then, (3.1) and (3.2) yield

$$
\begin{aligned}
\bar{F}_{T}(t) & =\bar{F}_{\{1,2\}}(t)+\bar{F}_{\{1,3\}}(t)-\bar{F}_{\{1,2,3\}}(t) \\
& =\bar{F}(t, t, 0)+\bar{F}(t, 0, t)-\bar{F}(t, t, t) \\
& =K\left(\bar{F}_{1}(t), \bar{F}_{2}(t), 1\right)+K\left(\bar{F}_{1}(t), 1, \bar{F}_{3}(t)\right)-K\left(\bar{F}_{1}(t), \bar{F}_{2}(t), \bar{F}_{3}(t)\right) \\
& =W\left(\bar{F}_{1}(t), \bar{F}_{2}(t), \bar{F}_{3}(t)\right),
\end{aligned}
$$

where

$$
W\left(x_{1}, x_{2}, x_{3}\right)=K\left(x_{1}, x_{2}, 1\right)+K\left(x_{1}, 1, x_{3}\right)-K\left(x_{1}, x_{2}, x_{3}\right) .
$$

In particular, if the components are independent, $K$ is the product copula (i.e., $K\left(x_{1}, x_{2}, x_{3}\right)=$ $x_{1} x_{2} x_{3}$ for $0 \leq x_{i} \leq 1$ and $\left.i=1,2,3\right)$, and

$$
W\left(x_{1}, x_{2}, x_{3}\right)=x_{1} x_{2}+x_{1} x_{3}-x_{1} x_{2} x_{3}=H\left(x_{1}, x_{2}, x_{3}\right) .
$$

Now, we are ready to state the first representation theorem for coherent systems with heterogeneous dependent components.

Theorem 3.1. If $T=\phi\left(X_{1}, \ldots, X_{n}\right)$ is the lifetime of a coherent system with component lifetimes $X_{1}, \ldots, X_{n}$ having survival copula $K$ and structure-dependence function $W$ with right-continuous increasing mean function $m_{W}$, then $T$ is equal in law to $T^{*}=\phi\left(Y_{1}, \ldots, Y_{n}\right)$ with identically distributed component lifetimes $Y_{1}, \ldots, Y_{n}$ with joint reliability function

$$
\mathrm{P}\left(Y_{1}>x_{1}, \ldots, Y_{n}>x_{n}\right)=K\left(\bar{G}_{W}\left(x_{1}\right), \ldots, \bar{G}_{W}\left(x_{n}\right)\right),
$$

where

$$
\bar{G}_{W}(t)=m_{W}\left(\bar{F}_{1}(t), \ldots, \bar{F}_{n}(t)\right) .
$$

Proof. Using the facts that $m_{W}$ is a right-continuous increasing function, $W(0, \ldots, 0)=0$, and $W(1, \ldots, 1)=1$, we note that $\bar{G}_{W}$ in (3.5) is a proper reliability function. Then, by using the fact that $m_{W}$ is the mean function of $W$ we obtain

$$
\bar{F}_{T}(t)=W\left(\bar{F}_{1}(t), \ldots, \bar{F}_{n}(t)\right)=W\left(\bar{G}_{W}(t), \ldots, \bar{G}_{W}(t)\right)=\bar{F}_{T^{*}}(t),
$$

where $T^{*}$ is the lifetime of the system under consideration when its component lifetimes are 
identically distributed with joint reliability function as in (3.4), with $T$ representing this same system with dependent component lifetimes governed by $K$ and $W$.

Note that when $K$ is the product copula (i.e., the components are independent), then the function $\bar{G}_{W}$ in (3.5) coincides with the function $\bar{G}$ in (2.3). Moreover, the function $m_{W}$ exists and it is an increasing continuous function when the copula is absolutely continuous with support $[0,1]^{n}$. As mentioned above, the existence and properties of the mean function $m_{W}$ are related to the concept of the diagonal section usually applied to copulas. The diagonal section associated to the function $W$ is defined as

$$
\delta_{W}(t)=W(t, \ldots, t) .
$$

If $W$ is the structure-dependence function associated with a coherent system then $\delta_{W}(t)$ is an increasing function with $\delta_{W}(0)=0$ and $\delta_{W}(1)=1$. If $\delta_{W}$ is a continuous function then

$$
\delta_{W}^{-1}(x)=\inf \left\{t: \delta_{W}(t)>x\right\}
$$

is an increasing right-continuous function. Then,

$$
m_{W}\left(x_{1}, \ldots, x_{n}\right)=\delta_{W}^{-1}\left(W\left(x_{1}, \ldots, x_{n}\right)\right)
$$

is a mean function of $W$. Moreover, $m_{W}$ is right continuous and increasing. Hence,

$$
\bar{G}_{W}(t)=m_{W}\left(\bar{F}_{1}(t), \ldots, \bar{F}_{n}(t)\right)
$$

is a proper reliability function with support included in $[0, \infty)$.

We can obtain additional signature representations when the survival copula $K$ is exchangeable. A copula (or a function) $K$ is exchangeable if

$$
K\left(x_{1}, \ldots, x_{n}\right)=K\left(x_{\sigma(1)}, \ldots, x_{\sigma(n)}\right)
$$

for any permutation $\sigma$ of the set $\{1, \ldots, n\}$. The new representation result is then as follows.

Theorem 3.2. If $T$ is the lifetime of a coherent system with signature $s=\left(s_{1}, \ldots, s_{n}\right)$ and with component lifetimes $X_{1}, \ldots, X_{n}$ having an exchangeable copula $K$ and structure-dependence function $W$ with right-continuous increasing mean function $m_{W}$, then

$$
\bar{F}_{T}(t)=\sum_{i=1}^{n} s_{i} \bar{G}_{i: n}(t),
$$

where $\bar{G}_{i: n}(t)=\mathrm{P}\left(Y_{i: n}>t\right)$ and $Y_{1: n}<\cdots<Y_{n: n}$ are the order statistics obtained from the random variables $Y_{1}, \ldots, Y_{n}$ with joint reliability function as in (3.4).

Proof. Note that from Theorem 3.1 we know that $T$ is equal in law to a coherent system with component lifetimes having the joint reliability function in (3.4). Also, if $K$ is exchangeable then the reliability function in (3.4) is also exchangeable. Moreover, we know that representation (3.6) holds in the case of systems with component lifetimes having joint exchangeable distributions; see [22]. Hence, we obtain the desired result.

As a consequence, we obtain the following ordering property.

Theorem 3.3. Let $T$ and $T^{*}$ be the lifetimes of two coherent systems with signatures $\boldsymbol{s}=$ $\left(s_{1}, \ldots, s_{n}\right)$ and $s^{*}=\left(s_{1}^{*}, \ldots, s_{n}^{*}\right)$, and with component lifetimes having the same exchangeable survival copula $K$. If $\mathbf{s} \leq_{\mathrm{st}} \mathbf{s}^{*}$, both systems satisfy the assumptions of Theorem 3.2, and the reliability functions $\bar{G}_{W}$ and $\bar{G}_{W^{*}}$ defined by (3.5) are such that $\bar{G}_{W} \leq \bar{G}_{W^{*}}$, then $T \leq_{\mathrm{st}} T^{*}$.

Proof. The proof follows from Theorem 3.2 and Theorems 6.B.14 and 6.B.23 of [28]. 
Using the preceding result, we can obtain similar representations based on series or parallel systems as follows.

Theorem 3.4. If $T$ is the lifetime of a coherent system with minimal and maximal signatures $\boldsymbol{a}=\left(a_{1}, \ldots, a_{n}\right)$ and $\boldsymbol{b}=\left(b_{1}, \ldots, b_{n}\right)$, respectively, and with component lifetimes $X_{1}, \ldots, X_{n}$ having an exchangeable copula $K$ and structure-dependence function $W$ with right-continuous increasing mean function $m_{W}$, then

$$
\bar{F}_{T}(t)=\sum_{i=1}^{n} a_{i} \bar{G}_{1: i}(t)
$$

and

$$
\bar{F}_{T}(t)=\sum_{i=1}^{n} b_{i} \bar{G}_{i: i}(t),
$$

where $\bar{G}_{1: i}(t)=\mathrm{P}\left(Y_{1: i}>t\right), \bar{G}_{i: i}(t)=\mathrm{P}\left(Y_{i: i}>t\right), Y_{1: i}=\min \left(Y_{1}, \ldots, Y_{i}\right), Y_{i: i}=$ $\max \left(Y_{1}, \ldots, Y_{i}\right)$, and the random variables $Y_{1}, \ldots, Y_{n}$ have the joint reliability function in (3.4).

The proof is similar to that of Theorem 3.2. It is proved in [22] that representations (3.7) and (3.8) hold in the case of systems with component lifetimes having joint exchangeable distributions.

The next representation result shows that the lifetime of a coherent system with dependent components is equal in law to the lifetime of a system with the same structure but with i.i.d. components.

Theorem 3.5. If $T$ is the lifetime of a coherent system with signature $s=\left(s_{1}, \ldots, s_{n}\right)$ and with component lifetimes $X_{1}, \ldots, X_{n}$ having structure-dependence function $W$, then

$$
\bar{F}_{T}(t)=\sum_{i=1}^{n} s_{i} \bar{G}_{i: n}(t),
$$

where $\bar{G}_{i: n}(t)=\mathrm{P}\left(Y_{i: n}>t\right)$ and $Y_{1: n}<\cdots<Y_{n: n}$ are the order statistics obtained from the i.i.d. random variables $Y_{1}, \ldots, Y_{n}$ with common reliability function

$$
\tilde{G}(t)=h^{-1}\left(W\left(\bar{F}_{1}(t), \ldots, \bar{F}_{n}(t)\right)\right),
$$

where $h$ is the reliability polynomial.

The proof is immediate from (3.3) and the definition of the reliability polynomial. This result proves that if in some inferential procedures we only have information about the lifetimes of the system (i.e., a sample $T_{1}, \ldots, T_{m}$ of system lifetimes), then we cannot distinguish between a system with dependent components and the system with the same structure and i.i.d. components with common reliability function $\tilde{G}$. For further comments on such identifiability issues, see [4].

Finally, it should be mentioned that all the preceding mixture representations can also be obtained for mixed systems.

\section{Acknowledgements}

The authors thank the anonymous reviewer for several helpful comments. JN, FJS, and NB acknowledge financial support for this research from the Ministerio de Ciencia y Tecnología and Fundación Séneca (under grants MTM2009-08311 and 08627/PI/08), the US Army Research 
Office (under grant W911NF-08-0077), and the Natural Sciences and Engineering Research Council of Canada and the research grant KSU-VPP-107 from King Saud University, Riyadh, Saudi Arabia, respectively.

\section{References}

[1] Agrawal, A. and Barlow, R. E. (1984). A survey of network reliability and domination theory. Operat. Res. 32, 478-492.

[2] Barlow, R. E. And Proschan, F. (1975). Statistical Theory of Reliability and Life Testing. Holt, Rinehart and Winston, New York.

[3] Belzunce, F., Franco, M., Ruiz, J.-M. and Ruiz, M. C. (2001). On partial orderings between coherent systems with different structures. Prob. Eng. Inf. Sci. 15, 273-293.

[4] Bhattacharya, D. and Samaniego, F. J. (2010). On estimating component characteristics from system failuretime data. Naval Res. Logistics 57, 380-389.

[5] Boland, P. J. (2001). Signatures of indirect majority systems. J. Appl. Prob. 38, 597-603.

[6] De Finetti, B. (1931). Sul concetto di media. Giornale Ist. Ital. Attuari 2, 369-396.

[7] Esary, J. D. and Proschan, F. (1963). Relationship between system failure rate and component failure rates. Technometrics 5, 183-189.

[8] Hardy, G. H., Littlewood, J. E. and Pólya, G. (1934). Inequalities. Cambridge University Press, London.

[9] Jasiński, K., NAvarro, J. and Rychlik, T. (2009). Bounds on variances of lifetimes of coherent and mixed systems. J. Appl. Prob. 46, 894-908.

[10] Khaledi, B.-E. and Shaked, M. (2007). Ordering conditional lifetimes of coherent systems. J. Statist. Planning Infer. 137, 1173-1184.

[11] Kochar, S., Mukerjee, H. and Samaniego, F. J. (1999). The "signature" of a coherent system and its application to comparisons among systems. Naval Res. Logistics 46, 507-523.

[12] Navarro, J. (2008). Likelihood ratio ordering of order statistics, mixtures and systems. J. Statist. Planning Infer. 138, 1242-1257.

[13] Navarro, J. and Hernandez, P. J. (2008). Mean residual life functions of finite mixtures, order statistics and coherent systems. Metrika 67, 277-298.

[14] Navarro, J. and Rubio, R. (2010). Comparisons of coherent systems using stochastic precedence. Test 19, 469-486.

[15] Navarro, J. ANd Rychlik, T. (2010). Comparisons and bounds for expected lifetimes of reliability systems. Europ. J. Operat. Res. 207, 309-317.

[16] Navarro, J. and Shaked, M. (2006). Hazard rate ordering of order statistics and systems. J. Appl. Prob. 43, 391-408.

[17] Navarro, J. ANd Shaked, M. (2010). Some properties of the minimum and the maximum of random variables with joint logconcave distributions. Metrika 71, 313-317.

[18] Navarro, J. AND SPIzzichino, F. (2010). Comparisons of series and parallel systems with components sharing the same copula. Appl. Stoch. Models Business Industry 26, 775-791.

[19] Navarro, J., Ruiz, J. M. and Sandoval, C. J. (2007). Properties of coherent systems with dependent components. Commun. Statist. Theory Meth. 36, 175-191.

[20] Navarro, J., Samaniego, F. J. and Balakrishnan, N. (2010). The joint signature of coherent systems with shared components. J. Appl. Prob. 47, 235-253.

[21] Navarro, J., Spizzichino, F. and Balakrishnan, N. (2010). Applications of average and projected systems to the study of coherent systems. J. Multivariate Anal. 101, 1471-1482.

[22] Navarro, J., Samaniego, F. J., Balakrishnan, N. and Bhattacharya, D. (2008). On the application and extension of system signatures in engineering reliability. Naval Res. Logistics 55, 313-327.

[23] Nelsen, R. B. (2006). An Introduction to Copulas, 2nd edn. Springer, New York.

[24] Rychlik, T. (2001). Projecting Statistical Functionals (Lecture Notes Statist. 160). Springer, New York.

[25] Samaniego, F. J. (1985). On closure of the IFR class under formation of coherent systems. IEEE Trans. Reliab. 34, 69-72.

[26] Samaniego, F. J. (2007). System Signatures and their Applications in Engineering Reliability (Internat. Ser. Operat. Res. Manag. Sci. 110). Springer, New York.

[27] Satyanarayana, A. and Prabhakar, A. (1978). New topological formula and rapid algorithm for reliability analysis of complex networks. IEEE Trans. Reliab. 27, 82-100.

[28] Shaked, M. And Shanthikumar, J. G. (2007). Stochastic Orders. Springer, New York.

[29] Shaked, M. and Suarez-Llorens, A. (2003). On the comparison of reliability experiments based on the convolution order. J. Amer. Statist. Assoc. 98, 693-702.

[30] Zhang, Z. (2010). Mixture representations of inactivity times of conditional coherent systems and their applications. J. Appl. Prob. 47, 876-885. 